\title{
Current Situation and Future of High Intensity Focused Ultrasound Therapy for Oncology
}

\section{Yadav Ajay Kumar ${ }^{1,2,3,4 *}$, Yuan Gengbiao ${ }^{1}$, Fan Yongzeng'2 , Haitao Ran ${ }^{2}$, Mork Chanrith $^{2}$, Jin Chengbing ${ }^{3}$ and Gnawali Suman ${ }^{4}$}

${ }^{1}$ Department of Nuclear Medicine, The Second Affiliated Hospital of Chongqing

Medical University, China

${ }^{2}$ Department of Ultrasound, The Second Affiliated Hospital of Chongqing Medical

University, China

${ }^{3}$ Department of HIFU, The Second Affiliated Hospital of Chongqing Medical

University, China

${ }^{4}$ Department of Radio-Diagnosis, Imaging and Nuclear Medicine, BP Koirala

Memorial Cancer Hospital, Bharatpur, Chitwan, Nepal

*Corresponding Author: Yadav Ajay Kumar, Department of Nuclear Medicine, The

Second Affiliated Hospital of Chongqing Medical University, China.
Received: May 09, 2021

Published: June 24, 2021

(C) All rights are reserved by Yadav Ajay

Kumar., et al.

\begin{abstract}
Day by day, High-intensity focused ultrasound (HIFU) therapy is becoming more familiar in oncology because it is non-invasive technique with fewer side effects and provides promising therapeutic results. Several HIFU therapy applications have approved by many approval authorities of several countries since last decade. In fact, now, HIFU is approved for the clinical treatment of uterine fibroid, bone pain palliation, ablation of the prostate carcinoma, pancreatic carcinoma, hepatocellular carcinoma (HCC), breast carcinoma, renal cell carcinoma (RCC) treatment of essential tremor as a first neurological application etc. Besides of this, HIFU is also broadly using in face and neck cosmetic reconstruction from last few decades and proving promising results. It is a novel, emerging, therapeutic modality that uses ultrasound waves, propagated through tissue media, as carriers of energy. HIFU has great potential for tumor ablation and the main mechanisms of HIFU ablation involve mechanical and thermal effects. In our review, we are trying to include an overview of basic principle of HIFU therapy, it's both types i.e., USG guided HIFU and MRI guided HIFU, their limitations and overview on future in oncology.
\end{abstract}

Keywords: HIFU; MRI Guided HIFU; USG Guided HIFU; Ablation

\section{Introduction and Background}

In medical field, lots diagnostic modalities previously only use as diagnostic use but after few decades it was developed as therapeutic use. Like X-ray, it was previously used only for diagnostic modalities i.e., general x-ray, fluoroscopic x-ray, mammography, computed tomography (CT) but later developed as therapeutic modalities also i.e., linear accelerator (Tele-therapy unit for oncology), X-Knife (Stereo-tactic Radio-surgery). The major differ- ent of these modalities for diagnostic and therapeutic is intensity (energy) of that modality i.e., in diagnostic procedures, minimum intensity that require to produce acceptable diagnostic images is extremely lower than therapeutic procedure.

Ultrasound is only using as diagnostic modalities and over the last decade. Later several High Intensity Focused Ultrasound (HIFU) based applications have received clinical approval in various countries in all over the world for therapeutic purpose. In

Citation: Yadav Ajay Kumar., et al. "Current Situation and Future of High Intensity Focused Ultrasound Therapy for Oncology". Acta Scientific Cancer Biology 5.7 (2021): 25-34. 
current situation, HIFU is approved for the clinical treatment of uterine fibroid, bone pain palliation, ablation of the prostate carcinoma, pancreatic carcinoma, hepatocellular carcinoma (HCC), breast carcinoma, renal cell carcinoma (RCC) treatment of essential tremor as a first neurological application etc.

In diagnostic ultrasound, Diverging or parallel ultrasound wave is used but for therapeutic purpose, converging ultrasound wave is used. The therapeutic use of High Intensity Focused Ultrasound (HIFU) is little bit similar to Stereo-tactic Radio-surgery but we use ultrasound wave in spite of radiation. HIFU is a non-invasive surgical technique that uses non-ionizing ultrasonic waves to heat tissue. The principle of HIFU is based on increasing the blood flow or lymph flow and destroys targeted tissue, such as tumors, through a number of mechanisms. As we know that ultrasound is not an ionizing radiation therefor there is no major undesirable biological effects on the non-targeted tissue occur as long as that the ultrasound energy is appropriately located and focused. Non-invasive nature of HIFU has attracted the attention of clinicians, investigators and companies from around the world as an innovative, interventional tool that might provide promising therapeutic result minor complication.

HIFU is very similar to ultrasonography imaging, even though low frequency and continuous waves are used rather than pulsed ultrasound waves are used to achieve the necessary thermal effect. Acoustic conversing lenses are used to accomplish the required intensity at the target tissue without harming the surrounding tissue. A similarity is using a conversing lens to focus sunlight; only the focal points of the conversing lenses have high intensity. While conventionally lenses have been used, phased displays are progressively common as they allow the focal position to be easily changed. In recent modalities, HIFU is combined with imaging techniques such as medical diagnostic ultrasound or MRI to enable guidance of the treatment and monitoring.

Over the past years, radiological imaging methods such as MRI, US and CT/X-ray have become an integral part of therapeutic interventions such as percutaneous and trans-arterial procedures to treat vascular and oncological diseases. Diagnostic Imaging guide treatment planning offers spatial guidance to reach the targeted cells and it helps real time monitoring of the intervention itself, thus ensuring increased safety and improved outcome. Furthermore, Main benefit of HIFU treatment response can be evaluated in the same session using appropriate diagnostic imaging protocols thereby providing a prognostic readout for efficacy.
In 1927, Wood., et al. explained the physical and biological effect of high intensity ultrasound wave and also described about the detrimental effects of high-intensity ultrasound on animals [1]. Mean a while, low intensity focused ultrasound (LIFU) has been adopted for physiotherapy i.e., joint pain, body pain treatment etc. In 1942, Lynn., et al. succeeded to made a focusing high-intensity ultrasound (HIFU) modality and recognized its thermo-destructive potency when used to an ex vivo cells sample as well as in vivo cells when targeting the brain trans-cranially in cats and dogs [2]. In 1955, Fry., et al. were successfully able to produce focal lesions within the central nervous system in several animal experiments and in his experiment the skull was removed to avoid extensive heating of the bone surface [3]. Nonetheless, for the long period of time, the clinical application of HIFU remained unsuccessful largely due to the lack of heating control and spatial targeting. In 1995, Stephan Madersbacher., et al. published a report on the clinical use of HIFU for prostate cancer [4] which was monitored by many supplementary clinical researches on its use on several body organs. In current situation HIFU is approved in Israel, Canada, Europe, Korea, Russia, USA, China and many more countries for treatment of different diseases as well as oncology. Our objective of this review is to focus on basic principle of HIFU therapy, its types, its uses, limitations and an overview on its future in medical field.

\section{Basic principle of HIFU}

Sound wave is mechanical energy that can only propagate through a medium in the form of waves. Sound can only transport energy from its source to another region as long as a medium is present. Ultrasound is a form of energy that has a higher frequency $(>20,000 \mathrm{~Hz}$ ) than the human ear can detect $(2020,000 \mathrm{~Hz}$; audible ranges).

In spite of the fact that it's utilize for helpful purposes originates before demonstrative applications by a few decades, ultrasound is most broadly known for its imaging capabilities. The entry of ultrasound (US) through tissue can lead to natural changes which will be reversible or irreversible. The organic importance of these impacts depends to a large extent on the vitality within the ultrasound bar and the objective of the introduction. At demonstrative levels, any changes are generally accepted to organically immaterial. For helpful ultrasound, useful cellular or useful impacts are intentionally looked for, whether these are at the cell layer level (e.g. transitory changes in penetrability to encourage medicate conveyance) or less unpretentious impacts such as the localized temperatures rises that are required to attain prompt warm rot in 
tall concentrated centered ultrasound (HIFU; this method is some of the time too alluded to as FUS).

The instruments by which ultrasound actuates such natural impacts in tissue can broadly be isolated into two classes - warm and no-thermal. As the title shows, warm impacts are those emerging from the temperature rise that comes about from the assimilation of ultrasound vitality because it passes through tissue. Non-thermal (or mechanical) impacts are those emerging either from the arrangement and activity of micron-sized bubbles within the field (acoustic cavitation) or from the stream of liquids actuated by the ultrasound weight wave (acoustic spilling).

The principal behind HIFU therapy is exceptionally straightforward. Just as example sunlight can be light fire to a dry leaf or piece of paper after brought to a tight focus using a magnifying glass, ultrasonic beams can be focused, resulting in energy concentration in the focal region which may be sufficient to induce immediate cell death. The focused ultrasound beam is produced from a transducer capable of delivering high power. The transducer parameters decide the shape and position of the central volume [5].

The HIFU therapy destroys only tissues lying within the focal region, clearing out encompassing structures undamaged; shaping a 'trackless lesion.' portrays a close up of the focal area. The concentrated surpasses that vital to initiate cytotoxic temperature levels as it were in this volume. Histology illustrates that the edge between live and dead cells is exceptionally sharp. HIFU hence offers the potential for particular destruction of tissue targets at profundity with no harm to overlying structures as shown in figure 1.

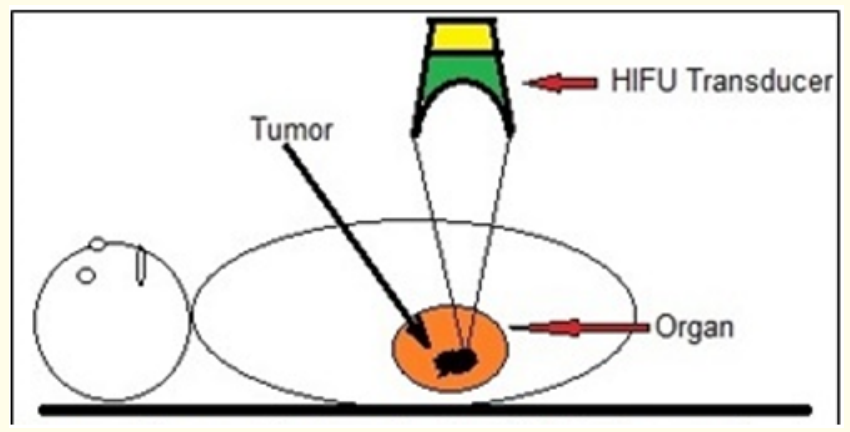

Figure 1: Principle of HIFU, an acoustic lens used to sound wave to a small point in the body that can be used to destroy targeted tissue. The sound propagates through many layers of the tissue. Because of the focal gain, only tissue at the focus is destroyed.

\section{Biological effects}

The elementary purpose of a HIFU medication may be the warm ablation of the picked focus. Ablation will be attained at those temperature is administered toward $56^{\circ} \mathrm{C}$ for $1 \mathrm{~s}$, or the warm equal [6]. These warm exposures bring about immediate cell demise from protein denaturation and harm on cytosolic also mitochondrial enzymes, furthermore starting with those framing from claiming histone complexes $[7,8]$. Histologically, the harm prompted is average from claiming coagulative corruption. Unpredictable cell division, furthermore sub-cellular transforms happen. These incorporate cells membrane, nucleic acid also mitochondrial work transforms [9]. Those progressions to cell layer smoothness, furthermore permanganic corrosive that bring about shortages starting with those expanded temperature prompt diminished cytoplasmic streaming furthermore impeded encouraged dissemination. Since indifferent dispersion may be unaffected, the net effect will be an amassing from claiming intracellular metabolites, deluge of extracellular fluids, Mobile swelling Also passing. Mitochondrial harm may be additionally a critical figure $[9,10]$. DNA amalgamation might a chance to be impeded toward that denaturation about non-histone proteins bringing about those restraints about union incitement by way proteins [11]. These sub-cellular impacts need aid inclined to be from claiming the vast majority importance in those HIFU lesion greatness edges the place the temperature arrived at is insufflate will make ablation, and the methodology of cell demise might a chance to be that's only the tip of the iceberg associated to that found clinched alongside "low" temperature hyperthermia.

Apoptosis is additionally seen in this tumor edge. Furthermore, with warm ablation, cell killing might make those outcomes about acoustic cavitation. It may be troublesome will separate histologically the middle of tissue impacts because of cavitation air pockets Also the individuals because of boiling, however following for acoustic outflows permits their separation. Tissue water bubbling occasions need aid went with toward capable of being heard sounds, while high back broadband and symphonious signs (of the drive) need aid trademark about acoustic cavitation. Clinched alongside general, intensities required on make bubbling temperatures throughout those short HIFU pulses surpass the limit level for prompting acoustic cavitation, and these two phenomena happen together. Histologically, the tissue may be seen will hold numerous voids the place the air pocket movement need made tears, principally over extracellular spaces. That excellent portrayal of the histological presence of HIFU lesion greatness is similarly as an "island" What's more "moat" [12]. This might make seen obviously utilizing hematoxylin and eosin (H\&E) staining. 
Focusing techniques of HIFU

There are various methods of focusing US waves and these have been another important issue. Self-focusing method is the simplest and cheapest technique as well as most accurate; a spherical ultrasound transducer (source). An ultrasound transducer fabricated according to focusing ultrasound beam fixed at the position determined from the geometrical stipulations of the transducer.

To pay compensation for its deficiency of flexibility, a flat ultrasound transducer with an interchangeable conversing acoustic lens system was devised. The acoustic lens allows changeable of focusing properties such as focal length and focal geometry. The main drawback of the lens system is that ultrasound waves undergo sound energy attenuation due to absorption by the lens [13]. In recent times, a phased array ultrasound transducer technique was implemented for HIFU ablation. By sending temporally different sets of electronic signals to each specific transducer component, this technology allows ultrasound beam routing and focusing, which can make to move a focal spot in practically any direction within physically allowed ranges. This technology is not only more multipurpose but also highly effective without any sonic attenuation than other model [14].

Image guided HIFU

High Intensity focused Ultrasound ablation therapy requires vigilant monitoring and so is usually achieved in combination with other diagnostic imaging techniques.

Pre-operative diagnostic imaging like prompt CT and MRI, are usually used to diagnose general factors of the target anatomy. On the other hand, real time diagnostic imaging is essential for safe and precise non-invasive ablation and therapy monitoring. Both MRI and Medical diagnostic ultrasound imaging have been used for guidance in FUS/HIFU treatment. These techniques are known as Magnetic Resonance guided Focused Ultrasound Surgery (MRgFUS) and Ultrasound guided Focused Ultrasound Surgery (USgFUS) respectively [15]. MRgFUS is a 3D imaging technique with High Intensity Focused Ultrasound (HIFU) which provides excellent soft tissue contrast and delivers information about temperature, thus allowing monitoring ablation. However, low frame rate makes these methods perform poorly in real time imaging and their high cost represents a main limitation to their use [16]. USgFUS, differently, is a $2 \mathrm{D}$ imaging technique in which, although no system to provide quantitative information on temperature has been commercially developed so far, several benefits are exploited, such as large frame rate (up to more than 1000 images per second), minimum cost and very few adverse health effects. In addition, treatment response evaluation can be monitor in real time through visual examination of hyper echoic changes in standard B-mode images [17]. Being MRgFUS a high-cost, labor intensive and slow imaging technique, recently, research groups are putting more efforts in improving USgFUS technology.

\section{Applications of HIFU}

HIFU is approved for the clinical treatment of various carcinomas since last few decades and proving promising results.

Uterine adenomyosis, fibroids and leiomyomas

The first approval of HIFU ablation from United States FDA to HIFU for symptomatic uterine fibroids was approved in October 2004 [18]. Studies have shown that HIFU is safe and effective, and that patients have continuous symptomatic relief is persistent for at least 2 years without the risk of difficulties involved in surgery or other more invasive approaches [19]. Up to $16-20 \%$ of patients will require additional treatment [20].

\section{Pancreatic carcinoma}

More than $80 \%$ of pancreatic cancer patients present advanced stage, and are inappropriate for surgical treatment. Available standard therapeutic options for advanced staged pancreatic carcinoma include either chemotherapy, radiation therapy or both in combination [21]. Due to not significant symptoms appears in early stage of pancreatic carcinoma, prognosis of pancreatic carcinoma is really extremely poor. Palliative treatment like pain palliation and supportive care are the principle aims of therapy to inoperable pancreatic carcinoma patients [22].

HIFU ablation has been used as primary and palliative treatment for pancreatic cancer [23]. Furthermore, HIFU ablation has been designed as an additional treatment with tumor debunking [21]. Various initial studies recommended that Focused Ultrasound ablative therapies may provide a survival benefit over palliative hospice care to advanced staged pancreatic cancer patients [24]. The main advantages of focused ultrasound ablation therapy are small recovery time and minimum side effects. One study reported that HIFU ablation guided by ultrasonography, this ablation tech- 
nique is highly feasible procedure to treat the solid tumors was concluded by a clinical study in which they included thirty-eight lesions in difficult positions inside liver and pancreas [25].

Another clinical study which included eighty-nine advanced staged pancreatic carcinoma patients who were treated with HIFU ablation therapy. This study concluded the median survival rate 26 months for stage II, 11.2 months for stage III, and 5.4 months for stage IV of patients with pancreatic carcinoma. Additionally, pain palliation was achieved up to approximately $80.6 \%$ of patients [26]. This above study highly recommended that clinical application of focused ultrasound ablation therapy for pain palliation in highly advanced staged pancreatic carcinoma patients [26]. There is another initial study of 8 patients with advanced staged pancreatic carcinoma, treated with ultrasound guided HIFU therapy. The authors reported that extremely sever back pain gone after focused ultrasound ablation therapy and there are no complications reported in all patients. HIFU ablation could be a safe procedure for patients with advanced pancreatic cancer [27].

Focused ultrasound ablation therapy is reported safe and feasible, highly capable to reduce cancer related abdominal pain, when locally advanced pancreatic cancer patients treated alone with HIFU or combined with chemotherapy [21]. HIFU ablation therapy could be one of the best treatment options for highly advanced staged pancreatic cancer patients if the long-term effective data is promising in large randomized clinical trials (RCT) [21]. Furthermore, new applications of HIFU therapy may improve outcomes of pancreatic cancer patients, through improving medicine transport to the targeted tissue (tumor) or producing self-immunity to both local and metastatic tumors [29]. Recently, research groups are putting more efforts on pancreatic Carcinomas.

\section{Prostate cancer}

The HIFU technique is also US FDA approved and could be an alternative to surgery especially for patients with comorbidity [30]. Careful patient selection is also imperative for prognosis. The patients with satisfactory pathologic Gleason score and lesser preHIFU PSA level appear to present better disease response [31]. Warmuth., et al. conducted a review on treatment of patients with prostate carcinoma with HIFU systems. 20 highly advanced staged prospective case series were acknowledged. The authors concluded that overall survival rate of patients was $83 \%$ and cancer related survival rate was $98 \%$ in 8 years [32].
Breast cancer

Breast-conserving therapy (BCT) is the current clinical management of localized breast carcinoma [33]. As advancement in technology, medical imaging and development in therapy modalities, the ablative technology of breast carcinoma has also progressed.

Noninvasive ablation (HIFU) of breast cancers could be an alternative option for small breast tumors [34]. There are so several questions arise in clinical applications of image guided percutaneous ablation of breast carcinoma. These questions and issues should be answer before promoting noninvasive percutaneous ablation can be approved as an alternative ablation of early breast cancer [35].

MRI imaging guided HIFU, which offers precision of anatomy and temperature mapping for ablation of a tumor without any bad effects to skin or subcutaneous tissue in the way of high intensity focused ultrasound wave, is absolutely non-invasive for breast carcinoma therapy. However, both technical and clinical issues have been resolved before MR-guided HIFU can be adopted as a routine clinical procedure (36).

Several clinical researches on HIFU ablation therapy for breast carcinoma were reported. Li., et al. conducted clinical studies on breast carcinoma treatment using HIFU guided by ultrasound imaging (US) and MRI during the period $2002-2010$. They included 173 breast cancer patients in their study with HIFU therapy and the tumor sizes varied from 0.5 to $6.0 \mathrm{~cm}$ diameter. Result of the study showed that complete ablation of tumor in MRI guided HIFU therapy or US guided HIFU therapy was 71\% (123173). On the other hand, complete necrosis rate of MIR guided HIFU and US guided HIFU were respectively 59\% (71173) and 96\% (50/52) in breast carcinoma patients (37).

There are many benefits of focused ultrasound ablation therapy, including protecting the real structure and function of breast, no bleeding, no scaring and no radiation. Even though, there are some limitations (disadvantages) of focused ultrasound ablation therapy for breast carcinoma treatment. Li S and Wu PH proposed three major problems with the HIFU therapy: (a) It is difficult to confirm whether ablation margin is free; (b) Recurrence concern exists in 5 multifocal or multi-centric breast cancers after HIFU treatment; (c) necrotic masses remaining in the breast after HIFU therapy could cause additional psychological burden to the patient (37). 
Hepatocellular carcinoma (HCC)

Gold standard therapeutic procedure for hepatocellular carcinoma is either liver transplantation or surgical resection. However, there are many challenges for extracorporeal ablations of liver tissue by HIFU, including movement of the liver due to respiration, penetration of beam though the ribs, and lengthy duration of ablation times due to large tumors $(38,39)$. HIFU ablation technology has been driven to overcome the challenges by several researches during the following decade.

Wu., et al. evaluated the efficiency using the combination of ultrasound guided HIFU modality and trans-catheter arterial chemoembolization (TACE) for fourth stage hepatocellular carcinoma (HCC) a treatment by randomized control clinical trial (RCT) study including fifty patients with Iva hepatocellular carcinoma (HCC). Median survival period was considerably longer in patients received combined treatment from both HIFU and TACE performed alone (11.3 months vs. 4 months; $\mathrm{p}=0.004)$. The 6 months survival rate of those patients who received combined treatment from both HIFU and TACE therapy was $80.4-85.4 \%$ whereas the survival rate of those patients who received treatment only from TACE therapy was $13.2 \%$ [p = 0.002] [40].

A clinical study demonstrated the efficacy and feasibility of focused ultrasound ablation therapy for locally advanced inoperable hepatocellular carcinoma. They included 116 primary HCC cases and 71 metastatic liver cancer cases treated by HIFU. Treatment response report after HIFU ablation therapy of that study was as follow; complete response (CR) and partial response (PR) were 55 patients (29.4\%) and 73 patients (39.0\%), respectively. The authors concluded that HIFU ablation therapy is feasible in hepatocellular carcinoma (HCC) and output of HIFU ablation may be improved by repeated treatment or increasing treatment times [41].

A research analyzed HIFU ablation technique for one hundred eighty-seven patients with unresectable hepatic carcinoma and concluded response rates $90.5 \%$ in left lobe cancer and $64.1 \%$ in right lobe cancer [42].

\section{Renal cell carcinoma}

For the clinical management of renal carcinoma, there are several invasive and non-invasive clinical treatment modalities including HIFU ablation therapy, surgical removal of tumor, radiofrequency ablation, and cryo-ablation [43]. Ablation treatment for renal carcinoma is highly advised to Surgical removable is gold standard and best option for localized renal cell carcinoma (RCC)at the present time. Till now, extracorporeal HIFU ablation for renal cells carcinoma (RCC) is well accepted but has to be considered an experimental approach [44]. A clinical research suggested that laparoscopic HIFU ablation for RCC could be possible, and achieved homogenous ablation with low associated morbidity [45].

Wu., et al. conducted clinical study with HIFU ablation therapy for renal cell carcinoma patients. The authors included total 13 patients in which 12 patients were locally advanced stage renal cell carcinoma (RCC) and 1 patient was gastro-intestinal carcinoma (colon cancer) with renal metastasis. Survival rate was demonstrated for all 13 patients. Among all thirteen patients, 7 patients deceased and median survival was 14.1 months. Out of 13 patients, six patients were alive at the time of paper writing and median survival was 18.5 months. It was summarized that High Intensity Ultrasound Therapy (HIFU) could be safe and feasible for advanced stage renal cell carcinoma patients [46].

Primary and secondary bone malignancy

Primary bone tumors are not common but mostly developed in children and young adult. Most common treatment options for primary bone malignancies are surgery and radiation therapy. Locally advanced and unresectable recurrence bone tumor is really challenging for curative treatment.

HIFU ablation technology has significant ability to ablate and inactive tumors. Limb sparing is a potential advantage of HIFU therapy for bone malignancies. Yu., et al. piloted a clinical trial research of HIFU ablation therapy for twenty-seven patients with local inoperable bone malignancies. The results showed 4 progression, 9 stable, 2 complete responses and 12 partial responses after HIFU therapy. For primary bone carcinoma patients, the tumor response evaluation rate achieved approximately $84.6 \%$; for patients with metastatic bone tumors, the tumor response rate was approximately $75.0 \%$. In this research, the authors suggested that HIFU ablation treatment appears to be effective in managing primary bone malignancies [47]. There are different views which were articulated for concern regarding survival; it contends HIFU is not designated for primary skeletal sarcoma therapy [48]. 
There were multicenter studies conducted to demonstrate efficacy of MRgFUS for palliative care in patients with bone metastases. That study reported that there were 18 patients out of 24 patients got significant pain relief i.e., $72 \%$ and there were no adverse effects occurred due to HIFU ablation therapy. It was concluded that MRgFUS could be one alternative choice for palliative treatment in the patients with painful bone metastases [49].

Thirteen patients with painful bone metastases were treated with MRgFUS ablation therapy to achieve pain palliation. Only 12 out of 13 patients treated MRgFUS ablation therapy. 10 out of 12 patients got significant pain relief and two patients died due to progressive disease. MRgFUS may be an alternative for bone metastasis pain relief [50].

\section{Trans-cranial HIFU therapy for brain tumor}

The major obstacle for HIFU technology in brain tumor ablation is cranial bone as the focused ultrasound field is troubled and most of the Ultrasound energy is absorbed by the skull bone. These problems have been overwhelmed for the development of adaptive focusing techniques and big sized phased array ultrasound transducers.

There was a trans-cranial clinical study done using MRgFUS ablation therapy for neuropathic pain palliation and brain tumor [51]. One another study concluded that MRgFUS ablation therapy could be used to temporarily interrupt the blood brain barrier [BBB] [52]. There was a pilot study included 15 patients who were treated with MRgFUS therapy for the treatment of medication refractory essential tremor and that study reported total tremor score improved from 54.9 to 24.3 [ $p=0.001$ ] [53].

Large RCT are necessary to evaluate the safety and efficacy of trans-cranial HIFU ablation therapy for cranial tumor and neuropathic pain.

\section{Benign and malignant thyroid carcinoma}

There are several studied are available in literatures concluded promising result of HIFU ablation for benign thyroid nodules specifically small nodules [54]. However, few pre-clinical studies are done for HIFU ablation for malignant thyroid carcinoma showed really good result [55]. Large size clinical studies are necessary to evaluate the safety and efficacy of HIFU ablation therapy malignant thyroid carcinoma.
Safety

Due to non-invasive, HIFU provides a very favorable safety profile. The most important risk is skin burn. To overcome this skin, burn risk, cooling devices and other safety techniques are used to guard the skin during the HIFU ablation treatment procedure.

\section{Post procedure side effects}

In the case of MRgFUS, patients are allowed to rest comfortably while the effects of any medication given are allowed to wear off. Occasionally, patients with fibroids may experience some abdominal pain or discomfort or menstrual-like cramping, and according to requirement, the physician provides directions or a prescription for pain relieving drugs after discharge (often only over-thecounter pain relief medication is required). There are common that most women are capable to return to work the next day or the day after.

\section{Limitations of HIFU}

As we know, common obstruction to ultrasound, like bone and gas, can interpose with uniform propagation of HIFU waves. Organ movement during HIFU procedures may lead to incomplete target ablation or collateral damage. The reflection of ultrasound causes similar effect by bone or gas-containing tissue. Therefore, tumors which are deeply seated in lungs are not ablated by HIFU. Hepatic cells carcinoma or any other tumors near hepatic dome are also most challenging due to movement of respiration may interfere with HIFU ablation propagation. To create same acoustic window for HIFU ablation, a dummy hydrothorax can be made. Improper acoustic pairing at the skin surface to transducer interface may also produce skin burns. The focusing of ultrasound through cranial bone is also one of the challenging advances that reduce the limitation of ultrasound reflection by bone. Since no needles are inserted inside the tumor during ablation, the tumor should always be within the range of the HIFU beam. This is one of the problems creating issue during ablation of target in organs affected by the respiratory movement. There are several researches are now ongoing to allow for automated motion tracking and beam refocusing to assure target tumor is always within the range of the therapeutic HIFU beam.

The accuracy of HIFU ablation may be accredited to the small lesion of necrosis from a separate sonication. However, such a limited 
necrosis volume makes HIFU treatment lengthy but new ablation techniques have been introduced to shorten the ablation time.

Future and promising HIFU research in oncology

There are lots of researches are ongoing to improve the treatment outcome, treatment of other oncological diseases and overcome the limitations.

In future, image guided HIFU could be give promising treatment protocol in oncology field with negligible side effects. As compare with MRgFUS, USgFUS is relatively cheaper and now widely using all over the world but USgFUS is two-dimensional imaging technologies where MRgFUS is three dimensional technologies. Due to 3Daccuracy, MRgFUS is giving more accuracy results than USgFUS. However, recently, research groups are putting more efforts in improving USgFUS technology. It can be concluded from recent clinical trials, pre-clinical studies and technology improvement day by day, HIFU will be one of the best options for oncology in near future.

\section{Conclusion}

Currently around the world, HIFU is of one of the interest to many physicians and research scientists. Present interest of HIFU ablation to clinical applications are mostly focused on therapy of body carcinoma, however many other potential clinical applications are currently under research. Due to significant nature of HIFU provides a non-invasive model for treatment of tumors that can mitigate many of the risks and side effects of current treatment modalities. However, HIFU ablation therapy has its limitations and there are several researches are ongoing to overcome that. It's our hope that advances in HIFU technology in the near future are likely to expand HIFU clinical applications.

\section{Acknowledgments}

All the authors are equally contributed to this article.

\section{Bibliography}

1. Wood RW and Loomis AL. "The physical and biological effects of high-frequency sound-waves of great intensity". Philosophical Magazine 4 (1927): 417-436.

2. Lynn JG., et al. "A New Method for the Generation and Use of Focused Ultrasound in Experimental Biology". Journal of General Physiology 26 (1942): 179-193.
3. Fry WJ., et al. "Ultrasonic lesions in the mammalian central nervous system". Science 122 (1955): 517-518.

4. Madersbacher S., et al. "Effect of high-intensity focused ultrasound on human prostate cancer in vivo". Cancer Research 55 (1995): 3346-3351.

5. TerHaar GR and Coussios C-C. "High intensity focused ultrasound: past, present and future". International Journal of Hyperthermia 23 (2007): 85-87.

6. Sapareto SA and Dewey WC. "Thermal dose determination in cancer therapy". International Journal of Radiation Oncology • Biology • Physics 10 (1984): 787-800.

7. Goldberg SN., et al. "Treatment of intra hepatic malignancy with radio-frequency ablation: pathologic correlation in 16 patients". American Journal of Roentgenology 170 (1998): 1023-1028.

8. Thompson S. "Pathologic analysis of photothermal and photochemical effects of laser-tissue interactions". Photochemistry and Photobiology 53 (1991): 825-835.

9. Farjado LF., et al. "Effects of hyperthermia on a malignant tumour”. Cancer 45 (1980): 613-623.

10. Yatvin MB and Cramp WA. "Role of cellular membranes in hyperthermia: some observations and theories reviewed". International Journal of Hyperthermia 9.2 (1993): 165-185.

11. Nikfarjam M., et al. "Mechanisms of focal heat destruction of liver tumours". Journal of Surgical Research 127 (2005): 208223.

12. Pond JB. "The role of heat in the production of ultrasonic focal lesions". Journal of the Acoustical Society of America 47 (1970): 1607-1611.

13. Vaezy S., et al. "Image-guided acoustic therapy". Annual Review of Biomedical Engineering 3 (2001): 375-390.

14. Hynynen K., et al. "MRI-guided focused ultrasound for local tissue ablation and other image-guided interventions". In: Wu J, Nyborg WL, eds. Emerging therapeutic ultrasound, 1st ed. Singapore: World Scientific Publishing (2006): 167-218.

15. Dubinsky., et al. "High-Intensity Focused Ultrasound: Current Potential and Oncologic Applications". American Journal of Roentgenology 190.1 (2008): 191-199.

16. Cafarelli A., et al. "A computer-assisted robotic platform for Focused Ultrasound Surgery: Assessment of high intensity focused ultrasound delivery". (2015): 1311-1314. 
17. Ebbini Emad S and TerHaar Gail. "Ultrasound-guided therapeutic focused ultrasound: Current status and future directions". International Journal of Hyperthermia 31.2 (2015): 7789.

18. Jump up to:a b Food and Drug Administration Approval, ExAblate ${ }^{2} 2000$ System - P040003.

19. Fennessy Fiona., et al. "Potential of minimally invasive procedures in the treatment of uterine fibroids: a focus on magnetic resonance-guided focused ultrasound therapy". International Journal of Women's Health 7 (2015): 901-912.

20. Stewart Elizabeth A., et al. "Sustained Relief of Leiomyoma Symptoms by Using Focused Ultrasound Surgery". Obstetrics and Gynecology 110 (2007): 279-287.

21. Wu F. "High intensity focused ultrasound: a noninvasive therapy for locally advanced pancreatic cancer". World Journal of Gastroenterology 20 (2014): 16480-16488.

22. Malietzis G., et al. "High-intensity focused ultrasound: advances in technology and experimental trials support enhanced utility of focused ultrasound surgery in oncology". British Journal of Radiology 86 (2013): 20130044.

23. Maloney E and Hwang JH. "Emerging HIFU applications in cancer therapy". International Journal of Hyperthermia 31 (2015): 302-309.

24. Keane MG., et al. "Systematic review of novel ablative methods in locally advanced pancreatic cancer". World Journal of Gastroenterology 20 (2014): 2267-2278.

25. Orsi F., et al. "High-intensity focused ultrasound ablation: effective and safe therapy for solid tumors in difficult locations". American Journal of Roentgenology 195 (2010): W245-252.

26. Xiong LL., et al. "Early clinical experience using high intensity focused ultrasound for palliation of inoperable pancreatic cancer". JOP 10 (2009): 123-129.

27. Wu F., et al. "Feasibility of US-guided high-intensity focused ultrasound treatment in patients with advanced pancreatic cancer: initial experience". Radiology 236 (2005): 1034-1040.

28. Jang HJ., et al. "Current and Future Clinical Applications of High-Intensity Focused Ultrasound (HIFU) for Pancreatic Cancer". Gut Liver 4 (2010): S57-61.

29. Fornage BD and Hwang RF. "Current status of imaging-guided percutaneous ablation of breast cancer". American Journal of Roentgenology 203 (2014): 442-448.
30. Di Mari A., et al. "Clinical use of high-intensity focused ultrasound in the management of different solid tumors". WCRJ 1 (2014): e295.

31. Alkhorayef M., et al. "High-Intensity Focused Ultrasound (HIFU) in Localized Prostate Cancer Treatment". Polish Journal of Radiology 80 (2015): 131-141.

32. Warmuth M., et al. "Systematic review of the efficacy and safety of high-intensity focused ultrasound for the primary and salvage treatment of prostate cancer". European Urology 58 (2010): 803-815.

33. Merckel LG., et al. "MR-guided high-intensity focused ultrasound ablation of breast cancer with a dedicated breast platform". Cardio Vascular and Interventional Radiology 36 (2013): 292-301.

34. Sabel MS. "Nonsurgical ablation of breast cancer: future options for small breast tumors". Surgical Oncology Clinics of North America Special 23 (2014): 593-608.

35. Fornage BD and Hwang RF. "Current status of imaging-guided percutaneous ablation of breast cancer". American Journal of Roentgenology 203 (2014): 442-448.

36. Merckel LG., et al. "MR-guided high-intensity focused ultrasound ablation of breast cancer with a dedicated breast platform". Cardio Vascular and Interventional Radiology 36 (2013): 292-301.

37. Li S and $\mathrm{Wu} \mathrm{PH}$. "Magnetic resonance image-guided versus ultrasound-guided high-intensity focused ultrasound in the treatment of breast cancer". Chinese Journal of Cancer 32 (2013): 441-452.

38. Sibille A., et al. "Extracorporeal ablation of liver tissue by highintensity focused ultrasound". Oncology 50 (1993): 375-379.

39. Malietzis G., et al. "High-intensity focused ultrasound: advances in technology and experimental trials support enhanced utility of focused ultrasound surgery in oncology". British Journal of Radiology 86 (2013): 20130044.

40. Wu F., et al. "Advanced hepatocellular carcinoma: treatment with high-intensity focused ultrasound ablation combined with transcatheter arterial embolization". Radiology 235 (2005): 659-667.

41. Chen L., et al. "High intensity focused ultrasound ablation for patients with inoperable liver cancer". Hepatogastroenterology 62 (2015): 140-143. 
42. Chen L., et al. "High intensity focused ultrasound ablation for patients with inoperable liver cancer". Hepatogastroenterology 62 (2015): 140-143.

43. Klatte T., et al. "The contemporary role of ablative treatment approaches in the management of renal cell carcinoma (RCC): focus on radiofrequency ablation (RFA), high-intensity focused ultrasound (HIFU), and cryoablation". World Journal of Urology 32 (2014): 597-605.

44. Ritchie RW., et al. "Laparoscopic high-intensity focused ultrasound for renal tumours: a proof of concept study". BJU International 107 (2011): 1290-1296.

45. Klatte., et al. "High-intensity focused ultrasound for the treatment of renal masses: current status and future potential". Current Opinion in Urology 19 (2009): 188-191.

46. Wu F., et al. "Preliminary experience using high intensity focused ultrasound for the treatment of patients with advanced stage renal malignancy". Journal of Urology 170 (2003): 22372240 .

47. Yu W., et al. "High-intensity focused ultrasound: Noninvasive treatment for local unresectable recurrence of osteosarcoma". Surgery on Oncology 24 (2015): 9-15.

48. Bielack SS., et al. "High-intensity focused ultrasound (HIFU) is not indicated for treatment of primary bone sarcomas". Cancer 117 (2011): 2822.

49. Liberman B., et al. "Pain palliation in patients with bone metastases using MR-guided focused ultrasound surgery: a multicenter study". Annals of Surgical Oncology 16 (2009): 140-146.

50. Catane R., et al. "MR-guided focused ultrasound surgery (MRgFUS) for the palliation of pain in patients with bone metastases-- preliminary clinical experience". Annals of Oncology 18 (2007): 163-167.

51. Jenne JW. "Non-invasive transcranial brain ablation with highintensity focused ultrasound". Frontiers of Neurology and Neuroscience 36 (2015): 94-105.

52. Ghanouni P., et al. "Transcranial MRI-Guided Focused Ultrasound: A Review of the Technologic and Neurologic Applications". American Journal of Roentgenology 205 (2015): 150159.

53. Elias WJ., et al. "A pilot study of focused ultrasound thalamotomy for essential tremor". The New England Journal of Medicine 369 (2013): 640-648.
54. Chung SR., et al. "Efficacy and safety of high intensity focused ultrasound (HIFU) for treating benign thyroid nodules: a systematic review and meta-analysis". Acta Radiology 61.12 (2020): 1636-1643.

55. Pouya Namakshenas and Afsaneh Mojra. "Microstructurebased non-Fourier heat transfer modeling of HIFU treatment for thyroid cancer". Computer Methods and Programs in Biomedicine 197 (2020): 105698.

\section{Volume 5 Issue 7 July 2021}

(c) All rights are reserved by Yadav Ajay Kumar., et al. 